\title{
DNA-Powered Stimuli-Responsive Single-Walled Carbon Nanotube Junctions
}

\author{
Giuseppe Amoroso, ${ }^{\dagger \ddagger}$ Qingyu Ye, ${ }^{+, \neq}$Keitel Cervantes-Salguero, ${ }^{\dagger}$ Gustavo Fernandez, ${ }^{\S}$ Alessandro Cecconello, ${ }^{+*}$ \\ and Matteo Palma ${ }^{+*}$ \\ + School of Biological and Chemical Sciences and Materials Research Institute, Queen Mary University of London, Mile End Road, \\ London, E1 4NS, UK
}

\Organisch-Chemisches Institut, Westfälische Wilhelms-Universität Münster, Corrensstrasse

40, 48149 Münster, Germany

\begin{abstract}
Reconfigurable stimuli-responsive molecular materials play an important role in the fabrication of nanoscale systems and devices. Here we report a bottom-up strategy for the reversible assembly of single-walled carbon nanotubes linear junctions in solution. The assembly/disassembly of the nanotubes can be controlled via the intrinsic responsiveness to different stimuli of sequence-specific DNA linkers forming the junctions.
\end{abstract}

Single-walled carbon nanotubes (SWCNTs) are nanomaterials of technological interest because of their nanoscale diameter and notable thermal, mechanical, and electrical properties. ${ }^{1,2}$ The construction of junctions between SWCNTs ${ }^{3-7}$ has been investigated for the fabrication of nano-electronic devices, ${ }^{8-11}$ including the use of SWCNTs as nanoelectrodes in molecular transport junctions and single-molecule investigations. ${ }^{4,12-17}$ Different methods have been explored for junction formation; ${ }^{3,6,7,18}$ in this context, the controlled assembly of linear (end-to-end) junctions through insolution approaches ${ }^{3,4,19-21}$ is particularly desirable for the low-cost (solution processable) fabrication ${ }^{22-24}$ of carbon nanotube-based devices. ${ }^{15,17}$ Nevertheless, while different technologically-relevant nanomaterials have been designed to respond to changes in the surrounding medium, ${ }^{25,26}$ the assembly of reversibly reconfigurable end-to-end SWCNT junctions in-solution has yet to be demonstrated; this would allow the facile fabrication of carbon nanotubebased stimuli-responsive molecular systems and devices.

In this regard, DNA is an interesting bio-polymer for the implementation of dynamic behaviour in nano-mechanical $\operatorname{architectures}^{19,21}$ due to its well-known chemistry (i.e. several modifications are available for the attachment of functionalities or nanoparticles) and the ability to act as an actuator that responds to a number of different stimuli, depending on its nucleotide sequence. Several studies reported the use of DNA as a molecular material for the construction of designed nano-objects ${ }^{27,28}$ or as a scaffold for the assembly of nanoparticles with precise spatial arrangement. ${ }^{29,30}$ Furthermore, various response mechanisms to different stimuli were demonstrated to be available for the activation of the dynamics of specific DNA sequences, including strand-displacement, ${ }^{31}$ ligand-aptamer complex formation, ${ }^{32}$ non-canonical base-pairing, ${ }^{33}$ and 3D motif formation (for example, G-quadruplex and imotif). ${ }^{34,35}$

Herein, we present a strategy for the controlled assembly of stimuliresponsive end-to-end SWCNT junctions in aqueous solution, employing DNA as a molecular linker: the dynamic behaviour of the junctions is controlled by the interaction of the DNA specific sequences with selected external stimuli. As a proof of concept, we fabricated linear assemblies of individual DNA-wrapped SWCNTs, by their end-functionalization with two partly complementary (vide infra) azido-modified single strands of DNA. The strands can hybridize to form a partial duplex under neutral $\mathrm{pH}$ conditions, thus forming SWCNT junctions. The formed junctions were disassembled by changing the $\mathrm{pH}$ of the solution from 7 to 5 , via the formation of a more stable i-motif structure at acidic $\mathrm{pH}$, hence destabilizing the DNA partial duplex linking the nanotubes. The individual SWCNTs were then subjected to neutral $\mathrm{pH}$, to restore the DNA duplex junctions, therefore demonstrating the reversibility of the system. Additionally, we extended the study demonstrating the construction of dynamic SWCNTs junctions that operate via the strand-displacement mechanism. The approaches developed in this study allowed us to obtain reconfigurable end-to-end SWCNT junctions, as indicated by morphological analysis [Atomic Force Microscopy (AFM)) and further supported by time-dependent fluorescence characterizations. The ability to switch the coupling between single SWCNT segments, forming individual junctions, is of great importance for their future implementation in nano- and molecular-electronic smart systems and devices. ${ }^{16,36-38}$

HiPco SWCNTs were used in this study and were dispersed aspurchased in aqueous solutions via DNA wrapping ${ }^{39,40}$ [see the Supporting Information (SI)]. The non-covalent wrapping of DNA around the nanotubes allows both the dispersion of the carbon nanotubes in biocompatible aqueous solutions and the protection of their side-walls, leaving the nanotube ends available for further functionalization. ${ }^{3,4,12,13}$ The starting DNA-wrapped SWCNTs had an average length of $131 \pm 72 \mathrm{~nm}$ as determined by AFM analysis (for a representative image, see Figure S1 in the SI). The uncertainty affecting the measured lengths of the nanotube nanostructures was estimated by the standard deviation (s.d.) values associated 
with the length distributions, as they appear in the AFM analysis; this is reported for all distributions (average length \pm s.d.).

The solution containing the DNA-wrapped SWCNTs was mixed with methanol to a water-methanol 1:20 final ratio; this solution was divided in two aliquots, and while DNA sequence (1) was added to the first one, DNA sequence (2) was added to the second aliquot (all the DNA sequences used in this study are listed in Table $\mathrm{S} 1$ in the SI). DNA sequences (1) and (2) are 5'-azidomodified oligonucleotide strands that include in their sequences a cytosine (C)-rich or guanine (G)-rich domain, respectively, that are partially complementary to one another. In order to covalently functionalize the ends of the nanotubes with DNA, the two solutions were UV-irradiated with a medium pressure immersion mercury lamp (Photochemical Reactors Ltd) to promote the formation of reactive nitrene groups, which in proximity of the free-SWCNTtips form aziridine photoadducts by a cycloaddition reaction ${ }^{41-43}$ (for a scheme of the reaction, see Scheme S1 in the Supporting Information). After centrifugal filtration to remove the unreacted excess of (1) and (2) and subsequent re-dispersion of the modified nanotubes in aqueous solution, (1)-functionalized SWCNTs, A1, and (2)-functionalized SWCNTs, A2, hybrids were obtained. This functionalization procedure generates SWCNTs that are covalently and region-specifically linked to the DNA strands: specifically, the SWCNTs carry single DNA molecules at the nanotube ends. ${ }^{4}$

In order to quantify the amount of azido-DNA reacted with $\mathbf{A 1}$ and A2, the concentration of DNA in the filtered solutions was estimated by spectrophotometry: readings were taken at a wavelength equal to $260 \mathrm{~nm}$. The initial concentration of both (1) and (2) in the solutions before irradiation with the mercury lamp was $2 \mu \mathrm{M}$, while the concentration of the removed, unreacted DNA in the filtered solution was found to be equal to $0.19 \mu \mathrm{M}$ and $0.17 \mu \mathrm{M}$ for (1) and (2), respectively (for the recorded spectra of the filtered solutions, see Figure S2 in the SI). Although most of the DNA seemed to have reacted, a direct quantification of the DNA coupled to the SWCNT ends is, at the moment, still under investigation. As the DNA wrapping protects the nanotube side-walls, leaving only the tube-ends available for further functionalization, this method provides a facile one-step strategy for the selective functionalization of SWCNT termini.

The aforementioned A1 and A2 were then employed as the building blocks for the formation of DNA-linked SWCNT junctions. In particular, (1) and (2) can hybridize at neutral $\mathrm{pH}$ forming a partial DNA duplex (calculated $\mathrm{Tm}=34^{\circ} \mathrm{C}$ ). In order to direct the assembly of SWCNTs junctions, A1 and A2 were mixed in equal amounts at $\mathrm{pH}=7$ in a solution containing $2 \mathrm{mM} 3-(\mathrm{N}-$ morpholino)propanesulfonic acid (MOPS) and $400 \mathrm{mM} \mathrm{NaCl}$; this leads to junction formation via DNA hybridization that results in the duplex $(\mathbf{1}) /(\mathbf{2})$, Scheme 1 .

\section{Scheme 1. SWCNTs junction formation}

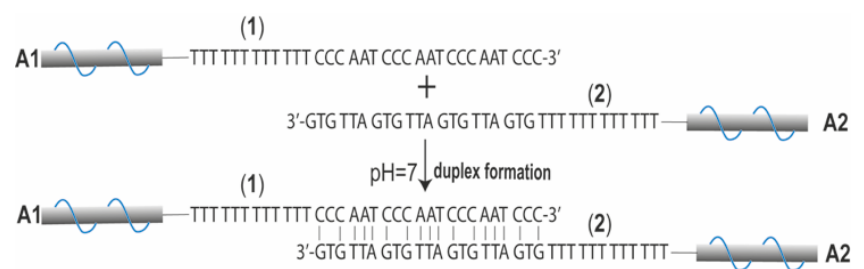

To demonstrate and monitor the assembly of the SWCNT junctions at $\mathrm{pH}=7$, dilute solutions were cast on mica and the substrates were imaged with AFM. We performed a statistical analysis of the average tube length; the average length of the SWCNTs junctions after one-hour incubation of $\mathbf{A 1}$ with $\mathbf{A 2}$ was found to be $184 \pm 97 \mathrm{~nm}$ (see Figure S3 for a representative AFM image and the related lengths distribution). In line with our previous SWCNT length analysis of junction formation, ${ }^{3,4}$ the increase in the SWCNTs average length from $131 \pm 72$ (Figure S1) to $184 \pm 97$ $\mathrm{nm}$ (Figure S3) indicates the formation of the junctions at $\mathrm{pH}=7$.

Both (1) and (2) contain different domains: a domain that forms the DNA partial duplex $(\mathbf{1}) /(\mathbf{2})$, and a thymine-rich region. By mixing the so-formed junctions with a biotin-modified adenine-rich (3) DNA sequence, which is complementary to the thymineregion, we could introduce biotin functionalities at the site of the junction formation. The presence of the biotin between the two SWCNT segments in the junctions was then exploited to label the junction site via the addition of streptavidin, that strongly and specifically interacts with biotin (for additional details on the biotinlabelled junction in the presence of streptavidin, see Scheme S2). This allowed us to then unequivocally locate the site of the junction formation via AFM imaging, hence further demonstrating the successful linking of A1 and A2. For this purpose, we drop-cast lowcoverage films of SWCNTs at $\mathrm{pH}=7$ on muscovite mica, then drop-cast a $500 \mathrm{nM}$ streptavidin solution, and after washing off the streptavidin excess with distilled water, the substrate was imaged by AFM. Figure 1 shows a representative AFM image and respective height profiles of a streptavidin-labelled SWCNT junction, corresponding to the expected nanotube and protein sizes: this confirms junction formation. It is worth mentioning that although at the junction point two biotin-labelled oligonucleotides are hybridized, it is assumed that only one streptavidin can bind to the site due to steric hindrance; this assumption is confirmed by AFM analysis of the junctions (see Figure S4). Although Figure 1 shows a straight junction, bent junctions are present in all our AFM images; this is clear from the AFM images presented in Figure S3 and in Figure S4. This is likely an effect of the physisorption of the SWNCT junctions from solution to surfaces, as previously observed by us and others. $^{3,19}$

a)

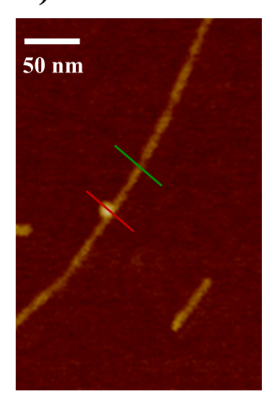

Figure 1. AFM characterization of the biotin-streptavidin labelled SWCNT junctions: a) representative topographical AFM image of a biotin-streptavidin labelled (1)/(2) DNA-SWCNT junction; b) AFM height analysis of the streptavidin-labelled SWCNT junction shown in (a).

At neutral $\mathrm{pH},(\mathbf{1})$ is available for duplex formation and consequently can hybridize with its complementary (2) forming nanotube junctions. Conversely, when the $\mathrm{pH}$ of the solution is acidic 
$(\mathrm{pH}=5),(\mathbf{1})$ forms an intercalated motif (i-motif structure), ${ }^{34,44,45}$ leading to the disassembly of the DNA-linked SWCNT junctions. This behaviour can then be exploited for the $\mathrm{pH}$-controlled assembly/disassembly of SWCNTs junctions. ${ }^{46}$

To demonstrate and monitor the disassembly of the SWCNT junctions as a function of $\mathrm{pH}$, we cast acidic solutions $(\mathrm{pH}=5)$ on mica and imaged the substrates with AFM. The average length of the SWCNTs junctions after changing the $\mathrm{pH}$ of the solution from 7 to 5 was found to decrease to $148 \pm 80 \mathrm{~nm}$ (see Figure S5), suggesting the separation of the junctions upon reducing the $\mathrm{pH}$ from 7 to 5 .

To further prove the successful $\mathrm{pH}$-controlled assembly/disassembly of SWCNTs junctions, time-dependent fluorescence analysis of the nanostructures was performed. We mixed A1 and $\mathbf{A 2}$ in equal amounts at $\mathrm{pH}=7$ in a solution containing $2 \mathrm{mM}$ MOPS and $400 \mathrm{mM} \mathrm{NaCl}$ as previously described to form the junctions, and added a quencher ( $\mathrm{Q}$-modified DNA sequence (4), complementary to the thymine-domain, that binds at the junction site. We then added to this solution a Cyanine 3 (Cy3)-modified DNA (5), which is partially complementary to (2). Scheme 2 shows the $\mathrm{pH}$-controlled mechanism of the SWCNT-DNA junction assembly/disassembly, in the presence of quencher and fluorophore labels (4) and (5), respectively.

At $\mathrm{pH}=7,(\mathbf{5})$ is free in solution as (2) is not available due to the junction formation via a more stable $(\mathbf{1}) /(\mathbf{2})$ duplex structure; when the $\mathrm{pH}$ is decreased from 7 to 5 , the disassembly of the junction frees (2) in A2, allowing it to hybridize with (5); this in turn results in the quenching of $\mathrm{Cy} 3$ due to its proximity to $\mathrm{Q}$ on the terminal ends of $\mathbf{A 2}$ (see Scheme 2). The sequences (4) and (5) were designed to position the $\mathrm{Q}$ and $\mathrm{Cy} 3$ functionalities at a specific separating distance. From geometric considerations of the DNA

\section{Scheme 2. pH-controlled system}

molecule, we calculated the distance separating $\mathrm{Cy} 3$ and the quencher in duplex structure $(\mathbf{2}) /(\mathbf{4}) /(\mathbf{5})$ to be equal to $1.7 \mathrm{~nm}$. This results in the efficient quenching of the fluorescence emission at $\mathrm{pH}=7$, while at $\mathrm{pH}=5$ the large separating distance between the Cy 3 and the quencher in the single-strand (5) and duplex $(\mathbf{2}) /(4)$, results in the inefficient quenching of the fluorophore. We monitored this change in fluorescence in real-time, by varying the $\mathrm{pH}$ from 7 to 5 in multiple cycles, and recording the fluorescence emission of $\mathrm{Cy} 3$, as shown in Figure 2. This allowed us to confirm the reversible $\mathrm{pH}$-dependent assembly/disassembly of the DNASWCNTs. ${ }^{47}$ Additionally, to confirm the selectivity of this $\mathrm{pH}$ system, we assembled nanotube junctions employing a dsDNA, as a linker, which is not affected by changes in $\mathrm{pH}$ : Figure $\mathrm{S} 6$ shows how the average length of the junctions does not vary from $\mathrm{pH}=7$ to $\mathrm{pH}=5$. It should be noted that the incremental drift in the fluorescence intensity is likely due to the slow aggregation and precipitation of the SWCNTs; this results in a time-dependent proportional decrease in optical density of the mixture, and increased collected emission from the fluorophore. Additionally, small deviations are observed, due to the manual operation and by small volume additions of concentrated acid/base.
$(5)$

5'-C AAT CAC A-CY3-3'

(4) 3'-AAAAAAAAAAAA

A1

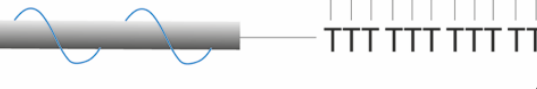

(1)<smiles>CC(C)(C)C</smiles>

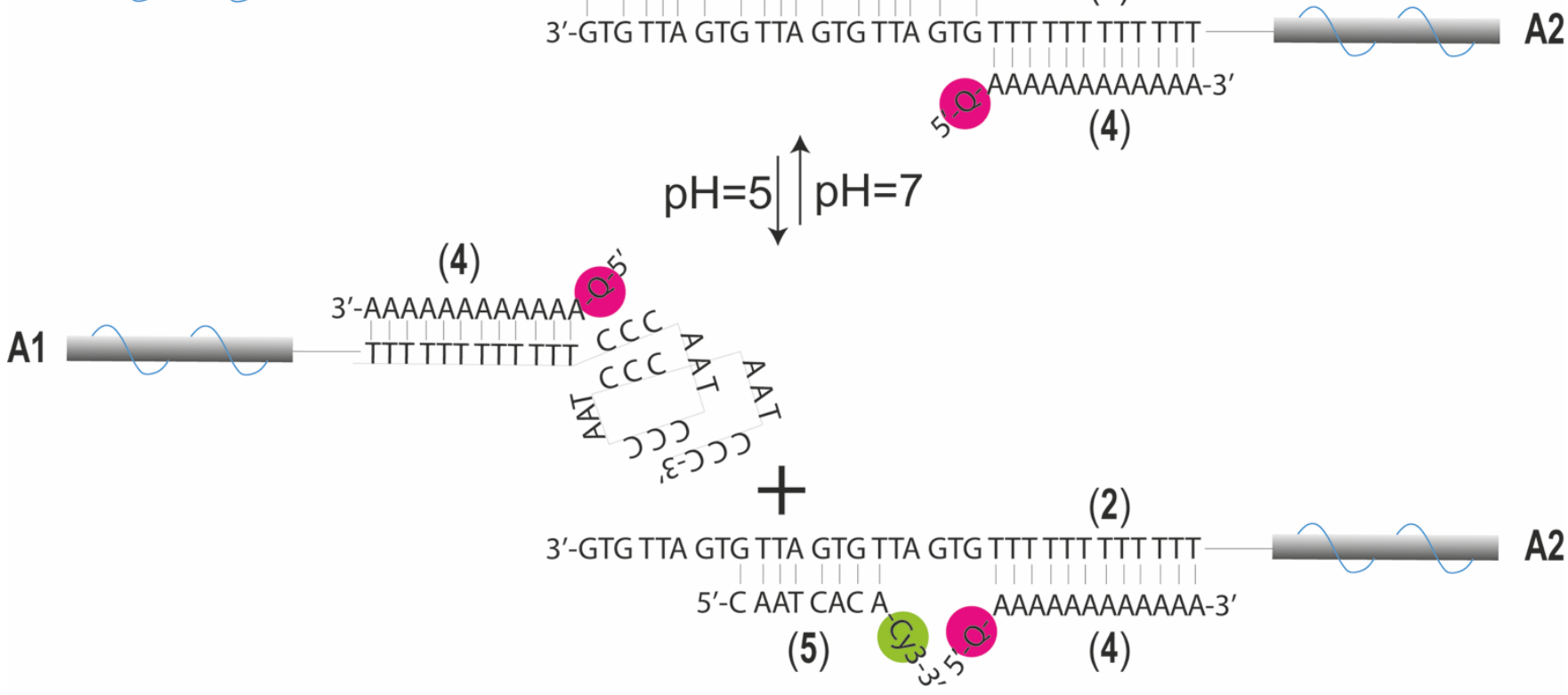




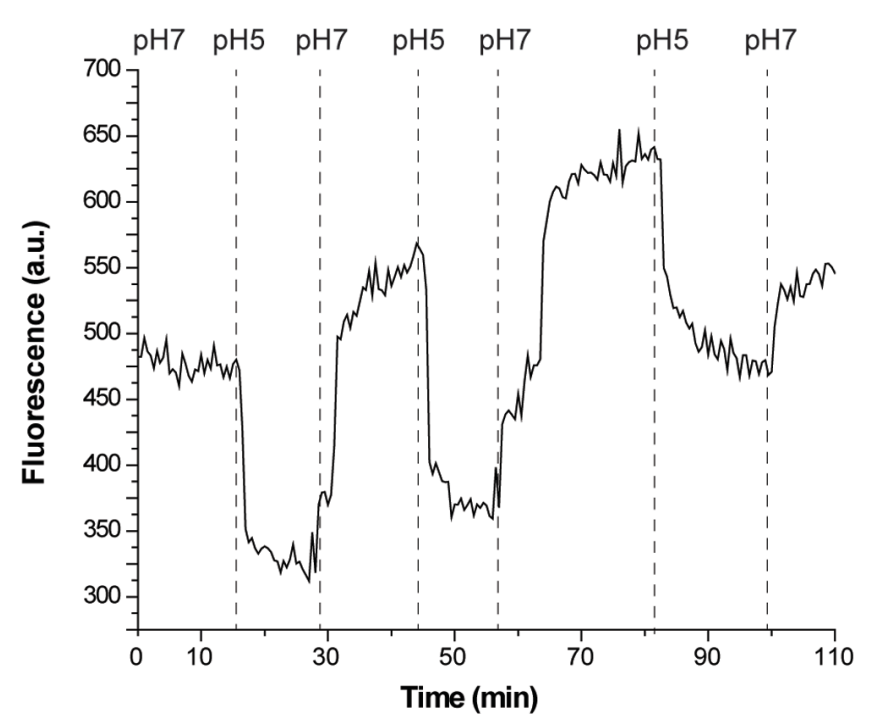

Figure 2. Real-time fluorescence changes of the $\mathrm{pH}$-controlled DNA-SWCNT junctions. The fluorescence emission of $\mathrm{Cy} 3$ is monitored while reversibly varying the $\mathrm{pH}$ between 7 and 5 , in multiple cycles. While high fluorescence emission corresponds to the formed DNA-SWCNT junctions at $\mathrm{pH}=7$ [where

Scheme 3. Strand-displacement system
Cy3-modified strand (5) is free in solution], low fluorescence emission corresponds to the separation of the DNA-SWCNT junctions at $\mathrm{pH}=5$ [where the $\mathrm{Cy} 3$-functionalized (5) is quenched by the quencher-modified strand (4)].

In order to explore the versatility of the approach developed here, we extended our studies by inducing the disassembly of SWCNT junctions with a different stimulus. A fuel DNA sequence (SD1), a DNA sequence that activates the disassembly process, was designed to be capable of destabilizing the DNA duplex forming the SWCNT junction, via strand-displacement. ${ }^{31,48}$ Upon addition of the designed fuel DNA strand (SD1) to the DNA-linked SWCNT junctions solution at neutral $\mathrm{pH}$, strand $(\mathbf{2})$ is displaced from duplex $(\mathbf{1}) /(2)$ by strand (SD1), resulting in the new duplex (1) $/($ SD1) and free strand (2). AFM images of disassembled SWCNT junctions by strand-displacement were taken after the addition of (SD1) to the SWCNT junctions: the disassembly was confirmed by statistical analysis of the average tube length, which was found to be $141 \pm 79 \mathrm{~nm}$ (see Figure S7), in accordance with the results obtained for the disassembled junctions at acidic $\mathrm{pH}$ $(148 \pm 80 \mathrm{~nm})$. To make this new system reversible, we introduced an anti-fuel DNA strand (SD2), a DNA sequence that re-activates the assembly process, which promotes the recovery of the assembly of $\mathbf{A 1}$ and $\mathbf{A 2}$ by a second strand-displacement event (Scheme 3). This was confirmed by time-dependent fluorescence measurements.

\section{(5)}

$5^{\prime}-$ C AAT CAC A-Cy3-3'

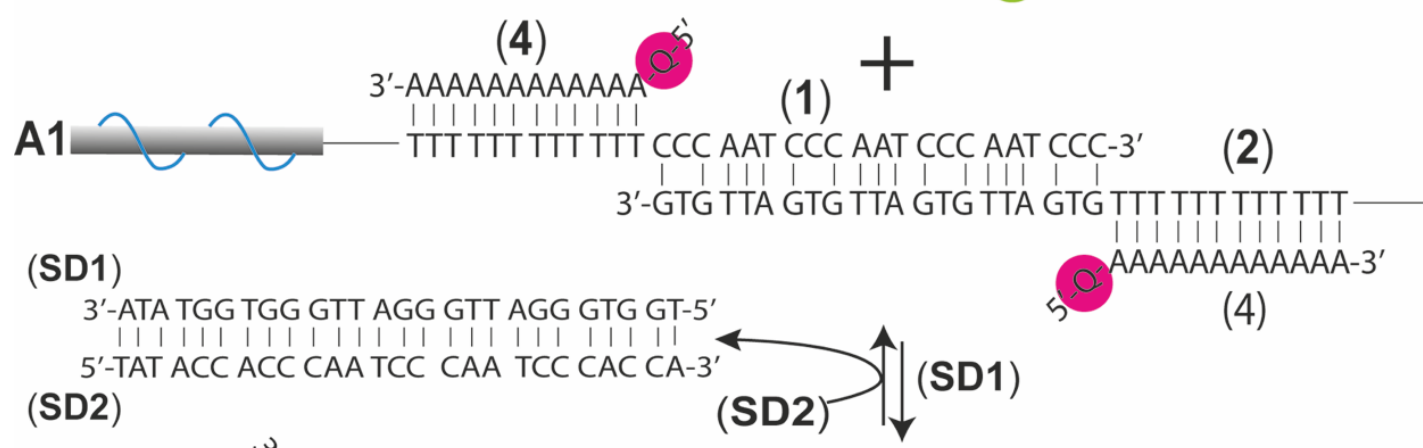

(SD1)

GTGGG TTA GGG TTA GGG TGGT-5' 要 CCC AAT CCC AAT CCC AAT CCC-3'

(4) 京次 齐众

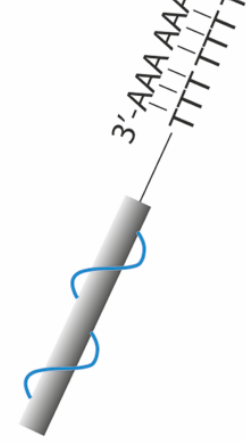

(1)

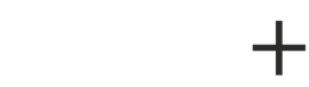

(2)

3'-GTG TTA GTG TTA GTG TTA GTG TTTTTTT TTT TTT 5'-C AAT CAC A

(5)

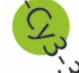
AAAAAAAAAAAA-3' 
Quencher-labelled $\mathbf{A 1}$ and $\mathbf{A 2}$ were mixed together at $\mathrm{pH}=7$ with (5); at neutral $\mathrm{pH} \mathrm{A1}$ and $\mathbf{A 2}$ form SWCNT junctions and the solution is characterized by a high fluorescence intensity due to the free Cy3-modified DNA (5) in solution. Upon addition of (SD1), the junction is separated and (5) binds to (2), resulting in the close proximity of $\mathrm{Cy} 3$ to $\mathrm{Q}$ and the low emission of fluorescence of $\mathrm{Cy} 3$. The following addition of (SD2) displaces (SD1) from the duplex $(\mathbf{1}) /($ SD1 $)$, resulting in the new duplex $($ SD1)/(SD2), and the restoration of the original SWCNT junction via the duplex (1)/(2). The concomitant release in solution of the fluorophoremodified strand (5) recovers the original high level fluorescence emission from $\mathrm{Cy} 3$ due to the large spatial separation from the quencher unit. Upon cyclic sequential addition in solution of (SD1) and (SD2) strands, the reversible assembly and disassembly of the SWCNT junctions is demonstrated by the low and high level of emission from Cy3-modified strand (5). While a high level of fluorescence characterizes the free fluorophore (formed junction), the close proximity of the fluorophore-quencher pair functionalities in the separated junctions is characterized by low fluorescence. Figure 3 shows the time-dependent fluorescence changes of the strand-displacement controlled SWCNT junction assembly/disassembly system, in the presence of quencher and fluorophore labels (4) and (5). To prove that the quenching effect of the carbon nanotubes on the fluorophores is not significant, in both the $\mathrm{pH}$-responsive and strand-displacement systems, we performed the same experiments in the absence of SWCNTs (see Figure S8 and Figure S9).

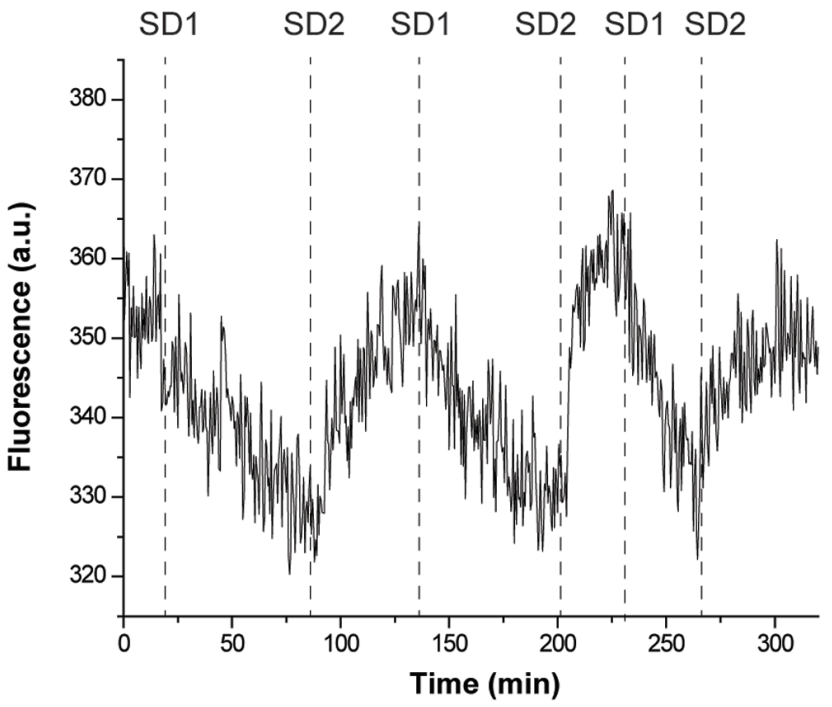

Figure 3. Real-time fluorescence changes of the stranddisplacement controlled DNA-SWCNT junctions in the presence of quencher-modified oligonucleotide (4) and Cy3-modified strand (5). Addition of (SD1) and (SD2) strands, in multiple cycles, results in the separation and recovery of the junction assembly, respectively. High fluorescence emission corresponds to the free Cy3-modified strand (5) in solution; while low fluorescence emission corresponds to the quenching of $\mathrm{Cy} 3-(5)$ upon separation of the DNA-SWCNT junctions.

In summary, we have presented a strategy for the controlled and reversible assembly of end-to-end SWCNT junctions under different stimuli. This was achieved by exploiting the molecular recognition of DNA complementary sequences, employed here as linkers. SWCNTs were covalently end-functionalized with azido-modified
ssDNA in a one-pot reaction, under UV light. SWCNTs assembly was driven by the hybridization between complementary DNA strands at the nanotubes termini at neutral $\mathrm{pH}$. Employing in the junction site a C-rich domain, the reversible assembly/disassembly of SWCNT junctions was controlled varying the $\mathrm{pH}$; this was confirmed by both AFM imaging and real-time fluorescence investigations. Additionally, we designed the system for the controlled and reversible assembly/disassembly of SWCNT junctions via stranddisplacement. This further demonstrates the versatility of the approach presented here, for the dynamic tuning of SWCNT junction formation via different stimuli. The strategy presented in this report is of interest for the fabrication of solution-processable and stimuliresponsive CNT-based systems, also of higher complexity. This holds great potential for the development of optoelectronic devices employing G-rich DNAs as active components, and sensing platforms via the use of oligonucleotide aptamer sequences, where the CNTs can be employed as switchable nanoelectrodes. ${ }^{16,36,49,50}$

\section{ASSOCIATED CONTENT}

Supporting Information. Experimental details, DNA sequences, scheme and explanation of the SWCNTs end-functionalization by photochemical reaction, spectra of DNA in the filtered solution after SWCNTs end-functionalization, additional AFM images, and statistical analysis of the SWCNTs length distribution after assembly/disassembly of the DNA-SWCNTs junctions.

\section{AUTHOR INFORMATION}

\section{Corresponding Author}

*alessandro.cecconello@gmail.com; *m.palma@qmul.ac.uk

\section{Author Contributions}

₹ These authors contributed equally. The manuscript was written through contributions of all authors. All authors have given approval to the final version of the manuscript.

\section{ACKNOWLEDGMENT}

The authors gratefully acknowledge financial support from the Air Force Office of Scientific Research under award FA9550-17-1-0179. G.A. is financially supported by the Materials Research Institute of Queen Mary University of London and the Westfälische Wilhelms University of Münster (WWU). A.C. is supported by a fellowship of the Federation of European Biochemical Societies (FEBS). Q.Y. is financially supported by the China Scholarship Council.

\section{ABBREVIATIONS}

SWCNT single-walled carbon nanotube; DNA deoxyribonucleic acid; AFM atomic force microscopy; MOPS 3-(Nmorpholino)propanesulfonic acid; Cy3 cyanine3.

\section{REFERENCES}

1. Baughman, R. H. Carbon Nanotubes--the Route Toward Applications. Science 2002, 297, 787-792.

2. Balandin, A. A. Thermal Properties of Graphene and Nanostructured Carbon Materials. Nat. Mater. 2011, 10, 569-581.

3. Palma, M.; Wang, W.; Penzo, E.; Brathwaite, J.; Zheng, M.; Hone, J.; Nuckolls, C.; Wind, S. J. Controlled Formation of Carbon Nanotube Junctions via Linker-Induced Assembly in Aqueous Solution. J. Am. Chem. Soc. 2013, 135, 8440-8443.

4. Zhu, J.; McMorrow, J.; Crespo-Otero, R.; Ao, G.; Zheng, M.; Gillin, W. P.; Palma, M. Solution-Processable Carbon Nanoelectrodes for Single-Molecule Investigations. J. Am. Chem. Soc. 2016, 138, 29052908.

5. McMorrow, J.; Freeley, M.; Palma, M. DNA-Wrapped Single-Walled 
Carbon Nanotube Assemblies. Ind. Eng. Chem. Res. 2017, 56, 53025308.

Zhen, Y.; Postma, H. W. C.; Balents, L.; Dekker, C. Carbon Nanotube Intramolecular Junctions. Nature 1999, 402, 273-276.

7. Fuhrer, M. S.; Nygård, J.; Shih, L.; Forero, M.; Yoon, Y. G.; Mazzoni, M. S. C.; Choi, H. J.; Ihm, J.; Louie, S. G.; Zettl, A.; McEuen P.L. Crossed Nanotube Junctions. Science 2000, 288, 494-497.

8. Park, H.; Afzali, A.; Han, S. J.; Tulevski, G. S.; Franklin, A. D.; Tersoff, J.; Hannon, J. B.; Haensch, W. High-Density Integration of Carbon Nanotubes via Chemical Self-Assembly. Nat. Nanotechnol. 2012, 7, 787-791.

9. Cao, Q.; Rogers, J. A. Ultrathin Films of Single-Walled Carbon Nanotubes for Electronics and Sensors: A Review of Fundamental and Applied Aspects. Adv Mat. 2009, 21, 29-53.

10. Park, S.; Vosguerichian, M.; Bao, Z. A Review of Fabrication and Applications of Carbon Nanotube Film-Based Flexible Electronics. Nanoscale 2013, 5, 1727-1752

11. Park, S.; Vosguerichian, M.; Bao, Z. A Review of Fabrication and Applications of Carbon Nanotube Film-Based Flexible Electronics. Nanoscale 2013, 5, 1727-1752.

12. Freeley, M.; Worthy, H. L.; Ahmed, R.; Bowen, B.; Watkins, D.; Macdonald, J. E.; Zheng, M.; Jones, D. D.; Palma, M. Site-Specific One-to-One Click Coupling of Single Proteins to Individual Carbon Nanotubes: A Single-Molecule Approach. J. Am. Chem. Soc. 2017, 139, 17834-17840.

13. Attanzio, A.; Sapelkin, A.; Gesuele, F.; van der Zande, A.; Gillin, W. P.; Zheng, M.; Palma, M. Carbon Nanotube-Quantum Dot Nanohybrids: Coupling with Single-Particle Control in Aqueous Solution. Small 2017, 13, 1603042.

14. Freeley, M.; Attanzio, A.; Cecconello, A.; Amoroso, G.; Clement, P.; Fernandez, G.; Gesuele, F.; Palma, M. Tuning the Coupling in Single-Molecule Heterostructures: DNA-Programmed and Reconfigurable Carbon Nanotube-Based Nanohybrids. Adv. Sci. 2018, 1800596.

15. Feldman, A.K.; Steigerwald, M.L.; Guo, X.; Nuckolls, C. Molecular Electronic Devices Based on Single-Walled Carbon Nanotube Electrodes. Acc. Chem. Res. 2008, 41, 1731-1741.

16. Guo, X. Single-Molecule Electrical Biosensors Based on SingleWalled Carbon Nanotubes Adv. Mater. 2013, 25, 3397-3408.

17. Xiang, D.; Wang, X.; Jia, C. Lee, T. Guo, X. Molecular-Scale Electronics: From Concept to Function. Chem. Rev. 2016, 116, 4318-4440.

18. Jin, C.; Suenaga, K.; Iijima, S. Plumbing Carbon Nanotubes. Nat. Nanotechnol. 2008, 3, 17-21.

19. Weizmann, Y.; Chenoweth, D. M.; Swager, T. M. Addressable Terminally Linked DNA- CNT Nanowires. J. Am. Chem. Soc. 2010, 132, 14009-14011.

20. Oruc, B.; Celik, S.; Hayat Soytas, S.; Unal, H. DNA Directed SelfAssembly of Single Walled Carbon Nanotubes into Three-Way Junction Nanostructures. ACS Omega 2018, 3, 4157-4162.

21. Wind, S.J.; Penzo, E.; Palma, M.; Wang, R.; Fazio, T.; Porath, D.; Rotem, D.; Livshits, G.; Stern, A. Integrating DNA with Functional Nanomaterials. Journal of Self-Assembly and Molecular Electronics (SAME) 2013, 1, 177-194.

22. Xu, X.; Clément, P.; Eklöf-Österberg, J.; Kelley-Loughnane, N.; Moth-Poulsen, K.; Chávez, J. L.; Palma, M. Reconfigurable Carbon Nanotube Multiplexed Sensing Devices. Nano Lett. 2018, 18, 41304135 .

23. Geier, M. L.; McMorrow, J. J.; Xu, W.; Zhu, J.; Kim, C. H.; Marks, T. J.; Hersam, M. C. Solution-Processed Carbon Nanotube Thin-Film Complementary Static Random Access Memory. Nat. Nanotechnol. 2015, 10, 944-948.

Brady, G. J.; Way, A. J.; Safron, N. S.; Evensen, H. T.; Gopalan, P.; Arnold, M. S. Quasi-Ballistic Carbon Nanotube Array Transistors with Current Density Exceeding Si and GaAs. Sci. Adv. 2016, e1601240.

25. Alivisatos, A. P. Semiconductor Clusters, Nanocrystals, and Quantum Dots. Science 1996, 271, 933-937.

26. Baker, S. N.; Baker, G. A. Luminescent Carbon Nanodots: Emergent Nanolights. Angew. Chem., Int. Ed. 2010, 49, 6726-6744.

27. Lu, C. H.; Cecconello, A.; Willner, I. Recent Advances in the Synthesis and Functions of Reconfigurable Interlocked DNA
Nanostructures. J. Am. Chem. Soc. 2016, 138, 5172-5185.

28. Rothemund, P. W. K. Folding DNA to Create Nanoscale Shapes and Patterns. Nature 2006, 440, 297-302.

29. Macfarlane, R. J.; Lee, B.; Jones, M. R.; Harris, N.; Schatz, G. C.; Mirkin, C. A. Nanoparticle Superlattice Engineering with DNA. Science 201 1, 334, 204-208.

30. Cecconello, A.; Besteiro, L. V.; Govorov, A. O.; Willner, I. Chiroplasmonic DNA-Based Nanostructures. Nat. Rev. Mater. 2017, 2, 17039 .

31. Zhang, D. Y.; Winfree, E. Control of DNA Strand Displacement Kinetics Using Toehold Exchange. J. Am. Chem. Soc. 2009, 131, $17303-17314$.

32. Hermann, T.; Patel, D. Adaptive Recognition by Nucleic Acid Aptamers. Science 2000, 287, 820-825.

33. Hu, Y.; Cecconello, A.; Idili, A.; Ricci, F.; Willner, I. Triplex DNA Nanostructures: From Basic Properties to Applications. Angew. Chemie 2017, 56, 15210-15233.

34. Gehring, K.; Leroy, J.-L.; Guéron, M. A Tetrameric DNA Structure with Protonated Cytosine-Cytosine Base Pairs. Nature 1993, 363, 561-565.

35. Siddiqui-Jain, A.; Grand, C. L.; Bearss, D. J.; Hurley, L. H. Direct Evidence for a G-Quadruplex in a Promoter Region and Its Targeting with a Small Molecule to Repress c-MYC Transcription. Proc. Natl. Acad. Sci. U.S.A. 2002, 99, 11593-11598.

36. Zhuravel, R.; Stern, A.; Fardian-Melamed, N.; Eidelshtein, G.; Katrivas, L.; Rotem, D.; Kotlyar, A.B.; Porath, D. Advances in Synthesis and Measurement of Charge Transport in DNA-Based Derivatives. Adv. Mater. 2018, 30, 1-11.

37. Guo, X.; Gorodetsky, A. A.; Hone, J.; Barton, J. K.; Nuckolls, C. Conductivity of a single DNA duplex bridging a carbon nanotube gap. Nat. Nanotechnol. 2008, 3, 163-7.

38. Wang, H.; Muren, N.B.; Ordinario, D.; Gorodetsky, A.A.; Barton, J.K.; Nuckolls, C. Transducing methyltransferase activity into electrical signals in a carbon nanotube-DNA device. Chem. Sci. 2011 3, 62-65.

39. Zheng, M.; Jagota, A.; Semke, E. D.; Diner, B. A.; McLean, R. S.; Lustig, S. R.; Richardson, R. E.; Tassi, N. G. DNA-Assisted Dispersion and Separation of Carbon Nanotubes. Nat. Mater. 2003, 2, 338-342.

40. Huang, X.; McLean, R. S.; Zheng, M. High-Resolution Length Sorting and Purification of DNA-Wrapped Carbon Nanotubes by Size-Exclusion Chromatography. Anal. Chem. 2005, 77, 6225-6228.

41. Lee, K. M.; Li, L.; Dai, L. Asymmetric End-Functionalization of Multi-Walled Carbon Nanotubes. J. Am. Chem. Soc. 2005, 127, 4122-4123.

42. Moghaddam, M. J.; Taylor, S.; Gao, M.; Huang, S.; Dai, L.; McCall, M. J. Highly Efficient Binding of DNA on the Sidewalls and Tips of Carbon Nanotubes Using Photochemistry. Nano Lett. 2004, 4, 8993.

43. Moghaddam, M. J.; Yang, W.; Bojarski, B.; Gengenbach, T. R.; Gao, M.; Zareie, H.; McCall, M. J. Azide Photochemistry for Facile Modification of Graphitic Surfaces: Preparation of DNA-Coated Carbon Nanotubes for Biosensing. Nanotechnology 2012, 23, 425503.

44. Wan, Y.; Liu, G.; Zhu, X.; Su, Y. PH Induced Reversible Assembly of DNA Wrapped Carbon Nanotubes. Chem. Cent. J. 2013, 7, 14-19.

45. Leroy, J. louis; Guéron, M.; Mergny, J. louis; Hélène, C. Intramolecular Folding of a Fragment of the Cytosine-Rich Strand of Telomeric DNA into an I-Motif. Nucleic Acids Res. 1994, 22, 16001606.

46. It should be noted that it was necessary to introduce mismatches in the DNA duplex forming the carbon nanotube junction in order to generate similar levels of thermal stability between the partial duplex $(\mathbf{1}) /(2)$ and the competing i-motif structure, which would be too unstable compared to the complete duplex $(\mathbf{1}) /(\mathbf{2})$, otherwise resulting in an inefficient separation of the junction at $\mathrm{pH}=5$.

47. It is worth mentioning that $\mathrm{Cy} 3$ fluorophore emission is insensitive to $\mathrm{pH}$ changes in the interval $\mathrm{pH}=5$ to $\mathrm{pH}=7$ thus in the described analysis the $\mathrm{Cy} 3$ emission intensity changes depend only on the average distance separating the fluorophore-quencher pair.

48. Seelig, G.; Soloveichik, D.; Zhang, D. Y.; Winfree, E. Enzyme-Free Nucleic Acid Logic Circuits. Science 2006, 314, 1585-1588. 
49. Jariwala, D.; Sangwan, V. K.; Lauhon, L. J.; Marks, T. J.; Hersam, M. C. Carbon Nanomaterials for Electronics, Optoelectronics, Photovoltaics, and Sensing. Chem. Soc. Rev. 2013, 42, 2824-2860. Lipomi, D. J.; Vosgueritchian, M.; Tee, B. C.-K.; Hellstrom, S. L.; Lee, J. A.; Fox, C. H.; Bao, Z. Skin-like Pressure and Strain Sensors Based on Transparent Elastic Films of Carbon Nanotubes. Nat. Nanotechnol. 2011, 6, 788-792. 

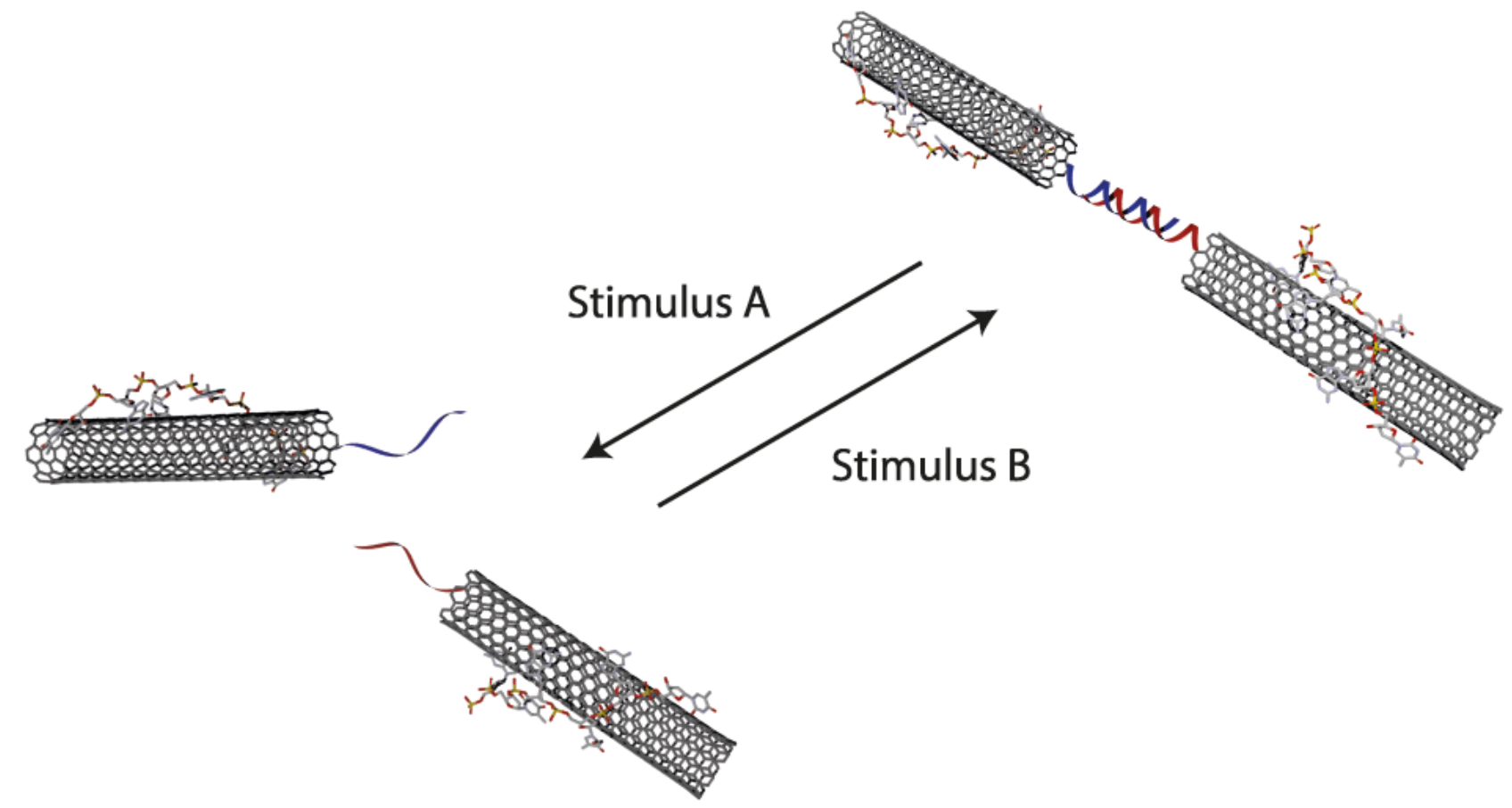